\title{
The 6-min walk test is useful for assessing prognosis in stable, ambulatory patients
}

New research has potentially provided clinicians with a convenient, easy-to-perform, less-costly, but similarly reliable, alternative to treadmill testing in the prognostic assessment of patients already diagnosed with stable coronary heart disease (CHD). As the study investigators point out, the potential replacement test "can be conducted with little equipment other than a hallway marked for distance and a stopwatch".

Dr Alexis Beatty and colleagues from the University of California, San Francisco, USA are interested in determining the impact of physical activity and exercise capacity on outcomes in patients with CHD. Thus, they set out to assess whether the 6-min walk test (6MWT) - a measure of functional exercise capacity that is used in the prognostic assessment of patients with heart failure or pulmonary hypertensioncould be useful in predicting cardiovascular events in patients with stable CHD.

The analysis involved a sample of patients enrolled in the Heart and Soul Study. All 556 assessed participants had stable CHD and completed the 6MWT and a treadmill test at baseline. Patients were followed up via annual telephone interview (with them or their proxy) for a median 8 years.

Compared with patients in the highest quartile of 6MWT distance at baseline, those in the lowest quartile had a substantially higher risk of heart failure, myocardial infarction, or death (unadjusted HR 4.29, 95\% CI 2.83-6.53, $P<0.001)$.
After adjustment for traditional risk factors and disease severity, each SD decrease in 6MWT distance was associated with a $30 \%$ increase in the rate of heart failure, myocardial infarction, or death. $6 \mathrm{MWT}$ distance was found to correlate with treadmill exercise capacity, and addition of 6MWT or treadmill results to the assessment of traditional risk factors resulted in similar improvements in category-free net reclassification $(39.3 \%$ and $39.6 \%$, respectively).

The investigators point out that, in the USA, the 6MWT is considered to be $\sim$ \$30 cheaper than standard treadmill stress testing used in patients with stable CHD undergoing prognostic assessment. When asked for his opinion on the recent findings, Dr Bernard Gersh from the Mayo Clinic, Rochester, MN, USA highlights that another benefit of the 6MWT is that it "can be done by physicians in office practice without the need to refer [patients with diagnosed stable CHD] to a facility that performs stress testing". He reminds us, however, that "for the diagnosis of CHD, formal stress testing is recommended".

Dr Beatty and colleagues suggest that, in addition to its use in assessing prognosis, the $6 \mathrm{MWT}$ could potentially be used as a tool to improve prognosis in patients with stable CHD. They hope that future studies will address this hypothesis. "Since we know that cardiac rehabilitation improves outcomes but is underutilized, finding additional tools for motivating patients

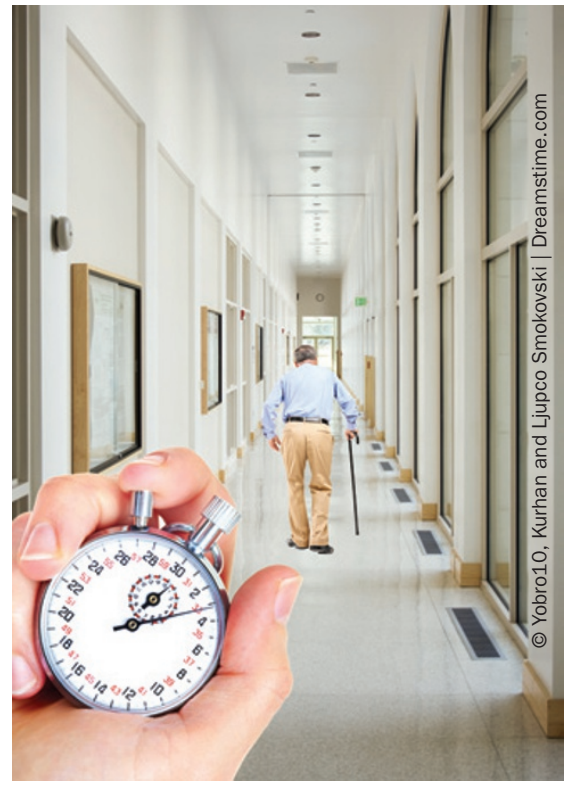

to meet physical activity goals is critical," highlights Dr Beatty. Dr David Nash, who wrote an editorial that accompanied the study report, agrees with the investigators and recommends that "physicians interested in improving their patients' level of fitness use the 6MWT as a means of getting the patient started on regular exercise. Once the patients become familiar with the ease and safety of the 6MWT, they can be encouraged to repeat [it] more frequently, even on a daily basis."

Bryony M. Mearns

Original article Beatty, A. L., Schiller, N. B. \& Whooley, M. A Six-minute walk test as a prognostic tool in stable coronary heart disease: data from the Heart and Soul Study.

Arch. Intern. Med. doi:10.1001/archinternmed.2012.2198 